\title{
A comparison of the photosynthetic radiation response of Scots pine shoots in direct and diffuse radiation
}

\author{
P. Oker-Blom ${ }^{1 *}$, H. Smolander ${ }^{2}$ and T. Lahti ${ }^{3}$ \\ 1 University of Helsinki, Department of Silviculture, Unioninkatu 40 B, $00170 \mathrm{Helsinki,}$ \\ 2 Finnish Forest Research Institute, Suonenjoki Research Station, SF-77600 Suonenjoki, and \\ 3 University of Helsinki, Botanical Museum, Unioninkatu 44, SF-00170 Helsinki, Finland
}

\section{Introduction}

The directional distribution of radiation incident on a coniferous shoot has been shown to have a large effect on the rate of shoot photosynthesis (e.g., Zelawski et al., 1973). In a multidirectional radiation field, the irradiance on the needle surface area of a shoot becomes more evenly distributed than in the case of a highly directional field, and the rate of photosynthesis per unit of intercepted radiation should logically be higher (cf. Oker-Blom, 1985). The aim of this study was to compare the rates of photosynthesis of Scots pine (Pinus sylvestris L.) shoots in diffuse and direct radiation and to test a shoot photosynthesis model based on the hypothesis that shoot photosynthesis can be expressed as the integrated response of the photosynthetic units of the shoot which are assumed to have an invariant photosynthetic light-response curve.

\section{Materials and Methods}

The material consisted of 9,1 yr old shoots collected from a young Scots pine stand. The net rate of photosynthesis of the excised shoots was measured in a direct and a diffuse (spherical) radiation field, using an open flow IRGAsystem (URAS $3 G$ ). The temperature in the assimilation chamber was $20^{\circ} \mathrm{C}$, ambient $\mathrm{CO}_{2}$ concentration was $340 \mathrm{ppm}$ and the air water vapor pressure deficit was $9 \pm 1$ mbar.

The distribution of radiation within each shoot was simulated using a Monte Carlo method (ct. Smolander et al., 1987) and using a model describing shoot geometry based on certain morphological characteristics of the shoot (cf. Oker-Blom et al., 1983). Using the simulated distributions and assuming the photosynthetic light curve for the photosynthetic unit to be a Blackman type curve (cf. Oker-Blom, 1985), shoot photosynthesis was calculated as the integrated response of the photosynthetic units. Parameters of the Blackman curve were estimated iteratively using the method of least squares to give the best fit between measured and calculated photosynthesis for the shoot in direct radiation.

\footnotetext{
"Present address: University of Georgia. School of Forest Resources, Athens, GA 30602, U.S.A.
} 


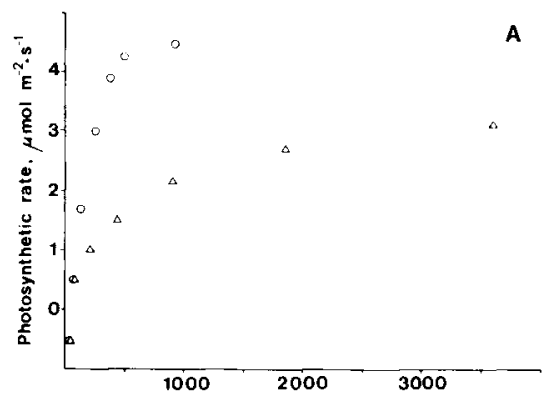

Horizontal photon irradiance, $\mu \mathrm{mol} \mathrm{m}^{-2} \cdot \mathrm{s}^{-1}$

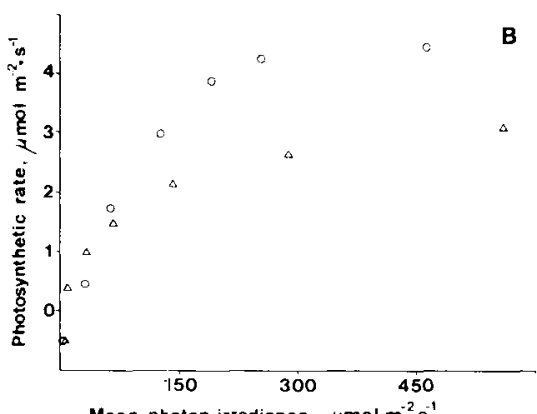

Mean photon irradiance, $\mu \mathrm{mol} \mathrm{m}^{-2} \cdot \mathrm{s}^{-1}$

Fig. 1. Rates of shoot photosynthesis in direct $(\Delta)$ and diffuse $(0)$ radiation, expressed as a function of (A) horizontal photon irradiance and (B) mean photon irradiance on the needie surface area.

In simulating the irradiance distributions, 2 different approaches were used. In the 1st case, the photosynthetic units of the shoot were represented by needle surface area elements, i.e., the distribution of irradiance on the needle surface area was simulated. In the 2 nd case, the photosynthetic units were represented by points within the needles and the irradiance (the photon field strength) at these points was simulated. The first approach is consistent with the assumption that the photosynthetic units are evenly distributed on the needle surface and that needles are optically black, i.e., there is no transmission of radiation within a needle. In the 2nd approach, the photosynthetic units are assumed to be uniformly distributed within the needle and the transmission of radiation was assumed to be an exponential function of the length of the photon pathway within the needle before reaching the point under consideration.

\section{Results}

Measured rates of photosynthesis of a shoot subjected to direct and diffuse radiation, respectively are shown in Fig. 1. When the radiation is expressed in terms of the (simulated) mean irradiance on the needle surface area (Fig. 1B), the rate of photosynthesis represents the photosyn-
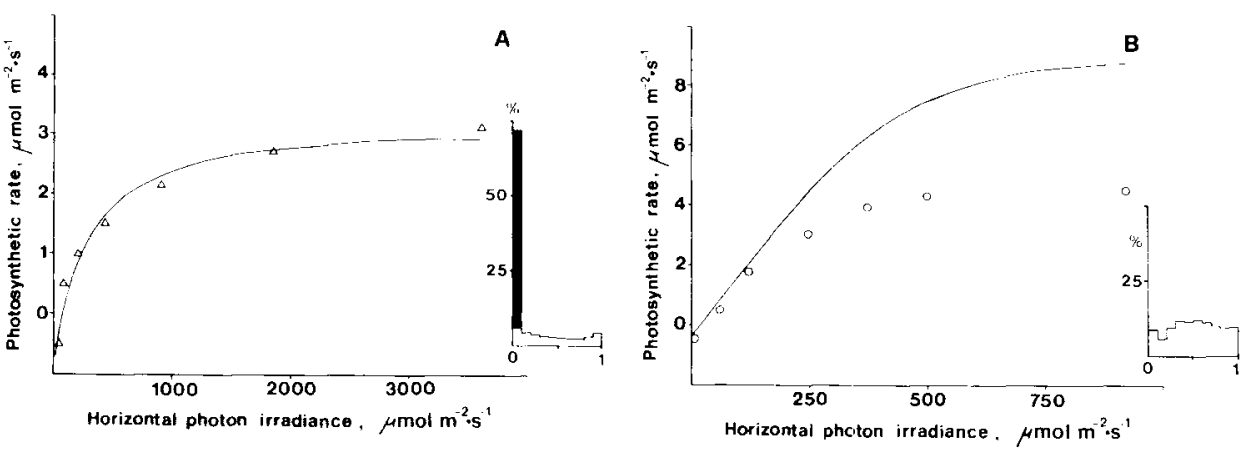

Fig. 2. Measured and calculated rates of shoot photosynthesis in (A) direct radiation and (B) diffuse radiation. Calculations were based on the simulated irradiance distributions on the needle surface area (insert: given as relative values, with 1 referring to horizontal irradiance) and the estimated photosynthetic light curve for a photosynthetic unit. 

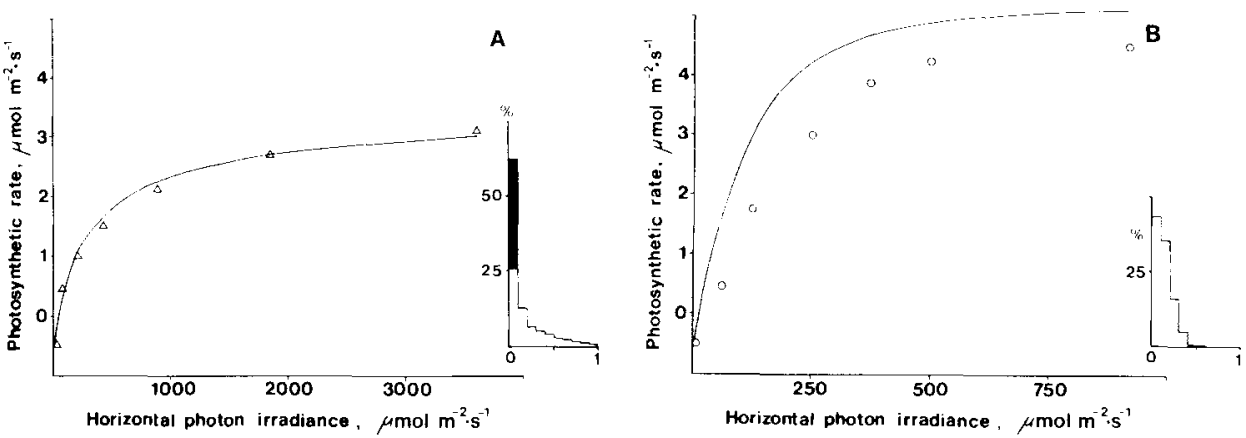

Fig. 3. Measured and calculated rates of shoot photosynthesis in (A) direct radiation and (B) diffuse radiation. Calculations were based on the estimated photosynthetic light curve for a photosynthetic unit and the simulated irradiance distributions within needles (insert: given as relative values, with 1 referring to horizontal irradiance in (A), and to photon field strength, i.e., $4 \times$ horizontal irradiance, in (B)).

thetic response per unit of intercepted radiation and the difference between the respective rates of photosynthesis result from differences in the distribution of radiation over the shoot.

In Fig. 2A, the photosynthetic rate of a shoot in direct radiation is calculated based on the simulated irradiance distribution on the needle surface area and a photosynthetic light curve with parameters a (initial slope) $=0.040$ and $P_{\mathrm{m}}$ (maximum rate $)=10.92 \mu \mathrm{mol}\left(\mathrm{CO}_{2}\right) \cdot \mathrm{m}^{-2} \cdot \mathrm{s}^{-1}$, estimated by the method of least squares to give the best fit to measured values. Using the same parameters and the simulated irradiance distribution in diffuse radiation, the rate of photosynthesis in the diffuse radiation field was predicted (Fig. $2 \mathrm{~B}$ ). The root mean square error of predicted rates in diffuse radiation varied between 1.45 and 3.65 and averaged $2.41 \mu \mathrm{mol}$ $\left(\mathrm{CO}_{2}\right) \cdot \mathrm{m}^{-2} \cdot \mathrm{s}^{-1}$ for the 9 shoots.

In Fig. 3A, the photosynthetic rate of a shoot in direct radiation is calculated using the distribution of radiation within the needles and a Blackman curve giving the best fit to measured values. The extinction coefficient along the path within the needle was taken as $3 \mathrm{~mm}^{-1}$, an arbitrary but representative value which corresponds to a transmission of $5 \%$ per $\mathrm{mm}$ of path length within the needle (cf. Gates et al., 1965). In Fig. 3B, the model is applied to diffuse radiation. The root mean square error of predicted rates by this 2 nd method varied between 0.31 and 1.58 and averaged $0.89 \mu \mathrm{mol}\left(\mathrm{CO}_{2}\right) \cdot \mathrm{m}^{-2} \cdot \mathrm{s}^{-1}$.

\section{Discussion}

Our results showed a clear difference between the rates of shoot photosynthesis in direct and diffuse radiation. When the radiation is expressed in terms of horizontal photon irradiance (Fig. 1A), the difference is exaggerated because, at an equal horizontal irradiance, the amount of intercepted radiation is many times great$e r$ in the spherical radiation field. In a direct radiation field, the amount of intercepted radiation, which is determined by the projected shoot area, has been shown to be the major component causing variation in the photosynthetic response (Smolander et al., 1987). Thus, much of the variation in photosynthesis caused by 
shoot structure and direction is eliminated when the rate of photosynthesis is expressed as a function of mean irradiance or, alternatively, on a projected shoot area basis. In the diffuse radiation field, however, the rate of photosynthesis per unit of intercepted radiation was still clearly higher (Fig. 1A), indicating that the more even distribution of radiation in the case of diffuse radiation is an important component, too.

In the direct radiation field, the fit of the measured rates to the estimated curve was rather good (Fig. 2A). When applied to shoot photosynthesis in a diffuse radiation field, however, the model gave clearly higher rates of photosynthesis than the measured ones (Fig. 2B). This deviation may be due to the assumption of optically black needles resulting in an overestimated difference between the irradiance distributions of photosynthetic units for direct and diffuse radiation. Therefore, an alternative model was developed which calculates the distribution of irradiance within the needles, assuming that the attenuation of radiation within a needle decreases exponentially. This model considerably improved the agreement between measured and calculated rates of shoot photosynthesis in diffuse radiation (Fig. 3).

In conclusion, it is proposed that a more invariant response of shoot photosynthesis to radiation may be obtained by expressing the radiation in terms of mean irradiance on the needle surface area, which partly eliminates the effect of shoot structure. The effect of radiation field geometry is, however, not completely offset by this method, which means that the relationship between intercepted radiation and photosynthesis depends upon, e.g., the shares of diffuse and direct radiation, respectively. For analyzing the effect of radiation field geometry, the method presented here was found to be promising.

\section{References}

Gates D.M., Keegan H.J., Schleter J.C. \& Weidner V.R. (1965) Spectral properties of plants. Appl. Opt. 4, 11-20

Oker-Blom P. (1985) Photosynthesis of a Scots pine shoot: simulation of the irradiance distribution and photosynthesis of a shoot in different radiation fields. Agric. For. Meteorol. 34, 31-40

Oker-Blom P., Kellomäki S. \& Smolander H. (1983) Photosynthesis of a Scots pine shoot: the effect of shoot inclination on the photosynthetic response of a shoot subjected to direct radiation. Agric. Meteorol. 29, 191-206

Smolander H., Cker-Blom P., Ross J., Kellomäki S. \& Lahti T. (1987) Photosynthesis of a Scots pine shoot: test of a shoot photosynthesis model in a direct radiation field. Agric. For. Meteorol. 39, 67-80

Zelawski W., Szaniawski R., Dybczynski W. \& Piechurowski A. (1973) Photosynthetic capacity of conifers in diffuse light of high illuminance. Photosynthetica 7, 351-357 